\title{
Pericoronary Adipose Tissue as Possible Supply Site and Storage for Lipoproteins in Human Coronary Artery
}

\section{Yasumi Uchida ${ }^{1,2 *}$ and Yasuto Uchida ${ }^{3}$}

${ }^{1}$ Japanese Foundation for Cardiovascular Research, Funabashi, Japan

${ }^{2}$ Department of Cardiology, Tokyo Jikei University School of Medicine, Tokyo, Japan

${ }^{3}$ Department of Cardiology, Tsukuba Memorial Hospital, Tsukuba, Japan

\begin{abstract}
It is a general belief that low-density lipoprotein (LDL) enters from the lumen into the vessel wall and oxidized (oxLDL) and acts as an important pro-atherogenic role in atherosclerosis and the anti-atherogenic substances such as high-density lipoprotein (HDL) and its component apolipoprotein A1 (ApoA1), also enters from the lumen. However, definite in vivo clinical evidence is lacking. We have demonstrated immunohistochemically that native oxLDL, HDL and ApoA1 co-saved in adipocytes in the majority of human pericoronary adipose tissue (PCAT) samples, and obtained marks that they are conveyed by either CD68(+) macrophages or vasa vasorum to the adjacent coronary. These results recommended the existence of before unrecognized storing and supply site of these proteins and that therapies directing the PCAT could be active in stopping human coronary atherosclerosis.
\end{abstract}

Keywords: Apolipoprotein A1; High-density lipoprotein; Human coronary plaques; Immunohistochemical staining; Low-density lipoprotein; Oxidized low-density lipoprotein; Pericoronary adipose tissue; Vasa vasorum

\section{Introduction}

It is a general belief that low-density lipoprotein (LDL) and monocytes enters the vascular wall from the lumen, the previous ends up noticeably oxidized low-density lipoprotein (oxLDL) and the last macrophages and accumulate oxLDL, after which oxLDL-containing macrophages develop foam cells, while producing collagen-degrading enzymes such as metalloproteases and collagenases which destroy collagen fibers and hypochlorous acid that destroy endothelial cells, resulting in vulnerable plaques [1-6]. It is also generally understood that anti-atherogenic substances like high-density lipoprotein (HDL) $[7,8]$ and its module apolipoprotein A1(ApoA1) [9-12] arrive the vascular intima from the vessel lumen either straight or thru vasa vasorum. Based on these generally thought mechanisms and by the plasma levels of lipoproteins and apolipoproteins as markers, lipid-lowering therapies are ordered in the clinical setting, but with narrow preventive effects on ischemic cardiovascular events.

\section{Hypothesis}

We hypothesized that there are further hitherto unrecognized storage site(s) and supply route(s) for pro-atherogenic and antiatherogenic lipoproteins and their components, which are not replicated in their plasma levels.

\section{OxLDL}

1-1. OxLDL in human PCAT (Pericoronary Adipose Tissue): By immunohistochemical techniques, we demonstrated that oxLDL is kept in adipocytes of epicardial PCAT, together cytoplasm and plasma membrane (Figures 1-3) [13].

1-2. Supply routes of oxLDL to adjacent coronary intima (plaque): OxLDL deposits showed either a diffuse (Figure 1B-1) or dotted pattern in intima (plaque) (Figure 1A-1) [13]. Double immunohistochemical staining showed that CD68(+) -macrophages contained dotted oxLDL deposits. CD68(+) -macrophages which contain oxLDL were present in interstitial spaces of PCAT (Figures 4B4B-2), adventitia, media and intima (Figure 2). They appeared to cross the adventitia, and external and internal elastic laminae, migrating from the PCAT to intima $[13,14]$.
1-3. OxLDL in plaques: In normal segments, the incidence was low, increased in thegrowth and mature stages and in the end stage of maturation gets decreased $[13,15]$.

1-4. OxLDL Co-localized with intimal vasa vasorum: Colocalization of vasa vasorum with diffuse oxLDL deposits was observed very frequently in the outer half (medial side) of the intima in plaques at growth stage than in normal segments, whereas more frequently in both the luminal (inner half) and medial (outer half) side of the intima in plaques at mature stage, indicating spread of oxLDL with development of vasa vasorum $[13,14]$. After these findings it was suggested that oxLDL was regularly carried to the intima by either neovascularized vasa vasorum or macrophages.

\section{HDL}

HDL co-depositions with oxLDL in PCAT: We found that HDL and oxLDL co-deposit in human PCAT [14]. HDL deposited in a diffuse pattern in the intima and dotted HDL deposits or HDL-containing CD68(+)-macrophages were not found (Figure 1C-1). Different to oxLDL, the HDL incidence in the coronary intima expanded further with plaque growth and expanded further with plaque maturation [14]. HDL co-localized with intimal vasa vasorum as with diffuse oxLDL deposits, suggesting that HDL in the PCAT was conveyed by neovascularized vasa vasorum to the intima.

\section{ApoA1}

ApoA1 Co-depositions with oxLDL in PCAT: ApoA1 placed in PCAT similarly to oxLDL and HDL. It deposited in either diffuse pattern or a dotted pattern in the intima (Figures 3B-1, 3B-2, 3C-1 and 3C-2) and contained in CD68(+)-macrophages or co-localized with an intimal vasa vasorum as with oxLDL. In typical normal coronary segments, the rate of

*Corresponding author: Yasumi Uchida, M.D, 2 -30 -17, Narashinodai, Funabashi, Japan, 274-0063, Tel: +81-47-462-2159; E-mail: uchida73@ta2.so-net.ne.jp

Received August 17, 2017; Accepted August 21, 2017; Published August 28 2017

Citation: Uchida Y, Uchida Y (2017) Pericoronary Adipose Tissue as Possible Supply Site and Storage for Lipoproteins in Human Coronary Artery. Mol Biol 6 : 195. doi: 10.4172/2168-9547.1000195

Copyright: (C) 2017 Uchida Y. This is an open-access article distributed under the terms of the Creative Commons Attribution License, which permits unrestricted use, distribution, and reproduction in any medium, provided the original author and source are credited. 

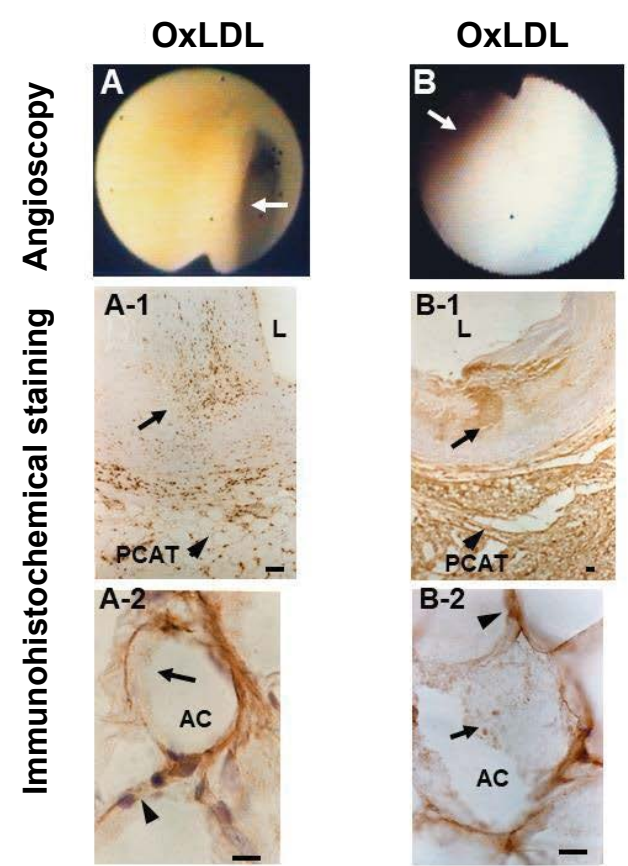

HDL
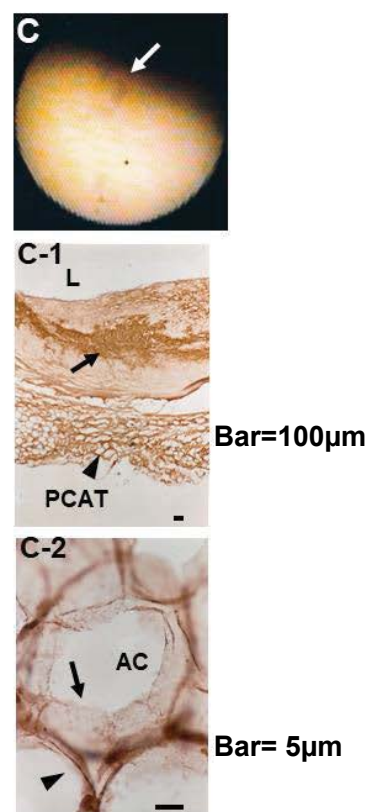

Figure 1: Localization and deposition patterns of oxidized low-density lipoprotein (OxLDL) and high-density lipoprotein (HDL) in human pericoronary adipose tissues (PCAT) and the coronary arterial wall. Yellow plaque by angioscopy [28] (arrow in A). Immunohistochemical staining shows, the plaque (arrow in A) contains dotted oxLDL deposits (arrow in A-1) and diffuse or dotted oxLDL deposits in the PCAT (arrowhead in A-1). Under magnification, oxLDL deposits not only in the cytoplasm (arrow in A-2) but also in the plasma membrane of adipocytes (arrowhead in A-2). White plaque by angioscopy [28] (arrow in B). Immunohistochemical staining shows diffuse oxLDL deposits in the intima (arrow in B-1) and PCAT (arrowhead in B-1). Under magnification, oxLDL deposits in the cytoplasm (arrow) and plasma membrane of the adipocytes (arrowhead in B-2). Yellow plaque by angioscopy28 (arrow in C). Immunohistochemical staining shows diffuse HDL deposits in the intima (arrow in C-1) and PCAT (arrowhead in C-1). Under magnification, HDL deposits in both the cytoplasm (arrow in C-2) and plasma membrane of the adipocytes (arrowhead in C-2). The cytoplasm is partially lost during preparation of the slide. White plaque by angioscopy [28] (arrow in D). Immunohistochemical staining shows, LDL deposits in the intima (arrow in D-1) but not in PCAT (arrowhead in D-1), under magnification, LDL is not observed in the cytoplasm or plasma membrane (arrowhead in D-2). AC: adipocyte, I: intima, M: media. Ad: adventitia. Bar in A-1 to D-1=100 $\mu \mathrm{m}$. Bar in A-2 to D-2=5 $\mu \mathrm{m}$. Partially modified with permission of Uchida et al. [14].

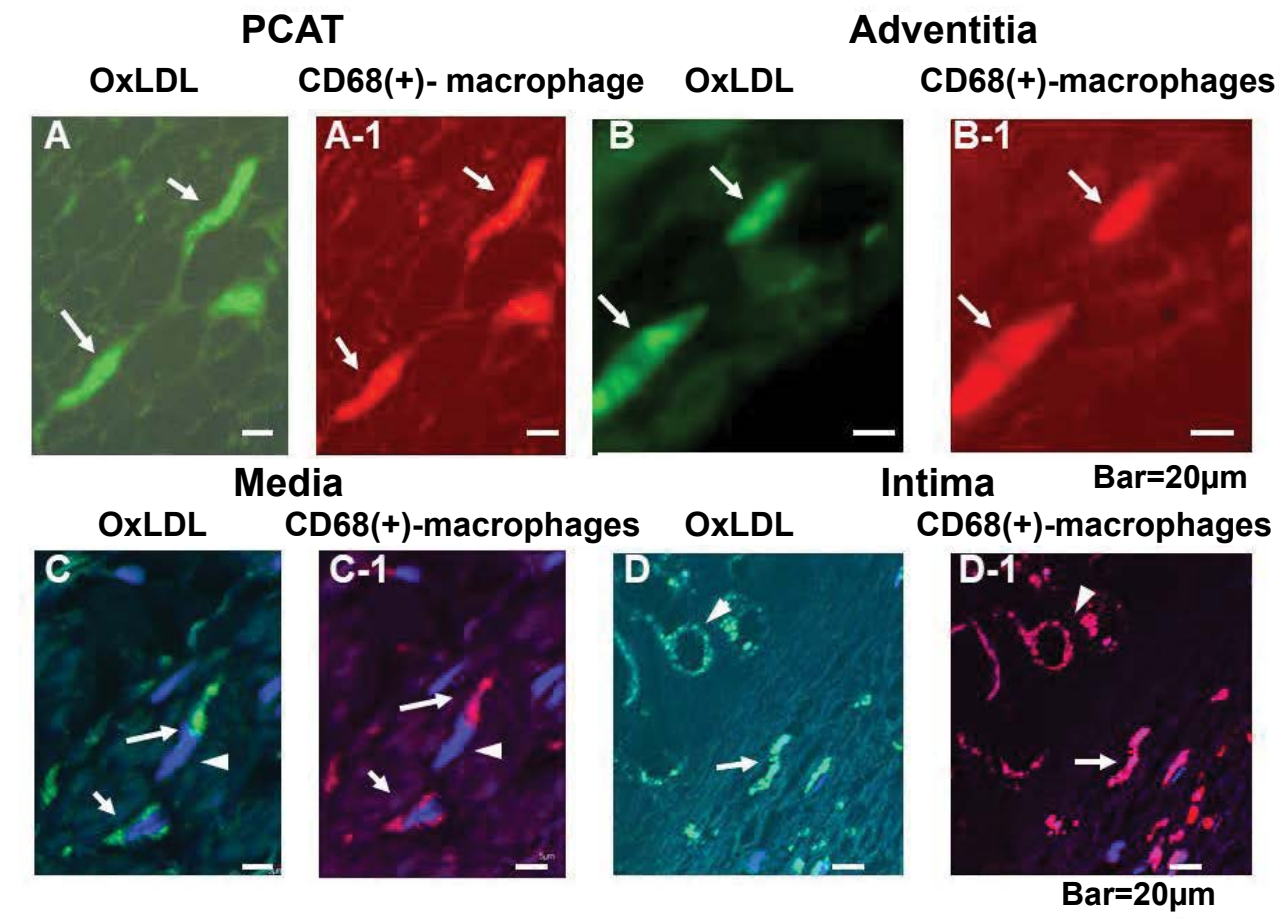

Figure 2: Oxidized Low-density Lipoprotein (OxLDL) containing CD68(+) macrophages. Ox-LDL is observed in CD68(+)-macrophages in PCAT (arrows in A, A-2), adventitia (arrows in B, B-1), media (arrows in C, C-1) and intima (arrows in D, D-1). Bar=5 $\mu$ m. Reproduced with permission of Uchida et al. [13]. 


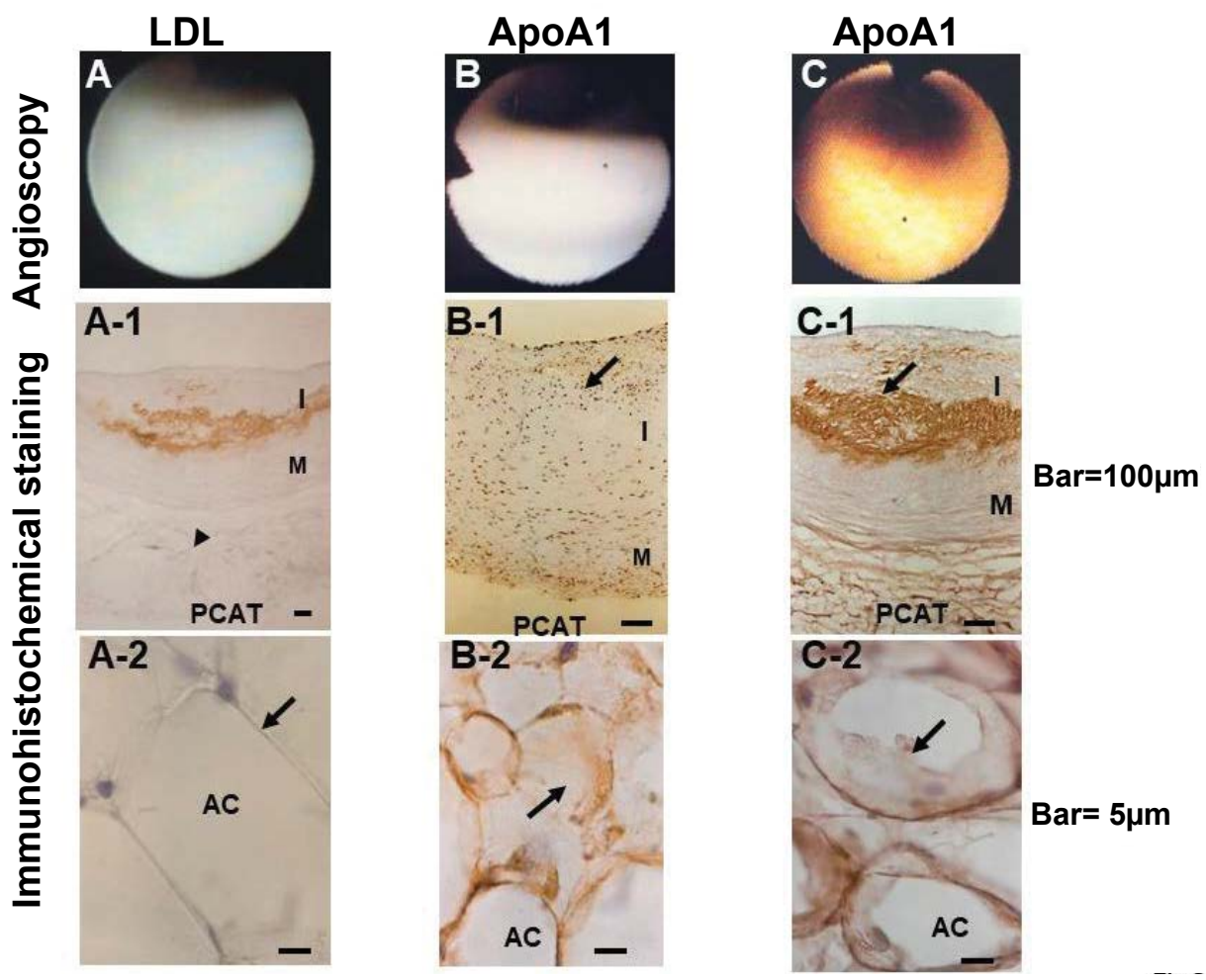

Figure 3: Localization and deposition patterns of low-density lipoprotein (LDL) and apolipoprotein A1 (ApoA1) in human pericoronary adipose tissues (PCAT) and the coronary arterial wall. White plaque on angioscopy [28] (arrow in A). Immunohistochemical staining shows, LDL deposits in the intima (arrow in A-1) but not in PCAT (arrowhead in A-1), under magnification, LDL is not observed in the cytoplasm or plasma membrane (arrowhead in A-2). White plaque on angioscopy (arrow in B). Immunohistochemical staining shows dotted ApoA1 deposits in the intima (arrow in B-1). Under magnification, diffuse ApoA1 deposits were found in PCAT (arrow in B-2). Yellow plaque on angioscopy (arrow in C). Diffuse ApoA1 deposits are observed in the plaque (arrow in C-1) and PCAT (arrow in C-2). AC: adipocyte, I: intima, M: media. Ad: adventitia. Bar in A-1 to $C-1=100 \mu \mathrm{m}$. Bar in A-2 to $C-2=5 \mu \mathrm{m}$. Partially modified with permission of Uchida et al. [14,16].
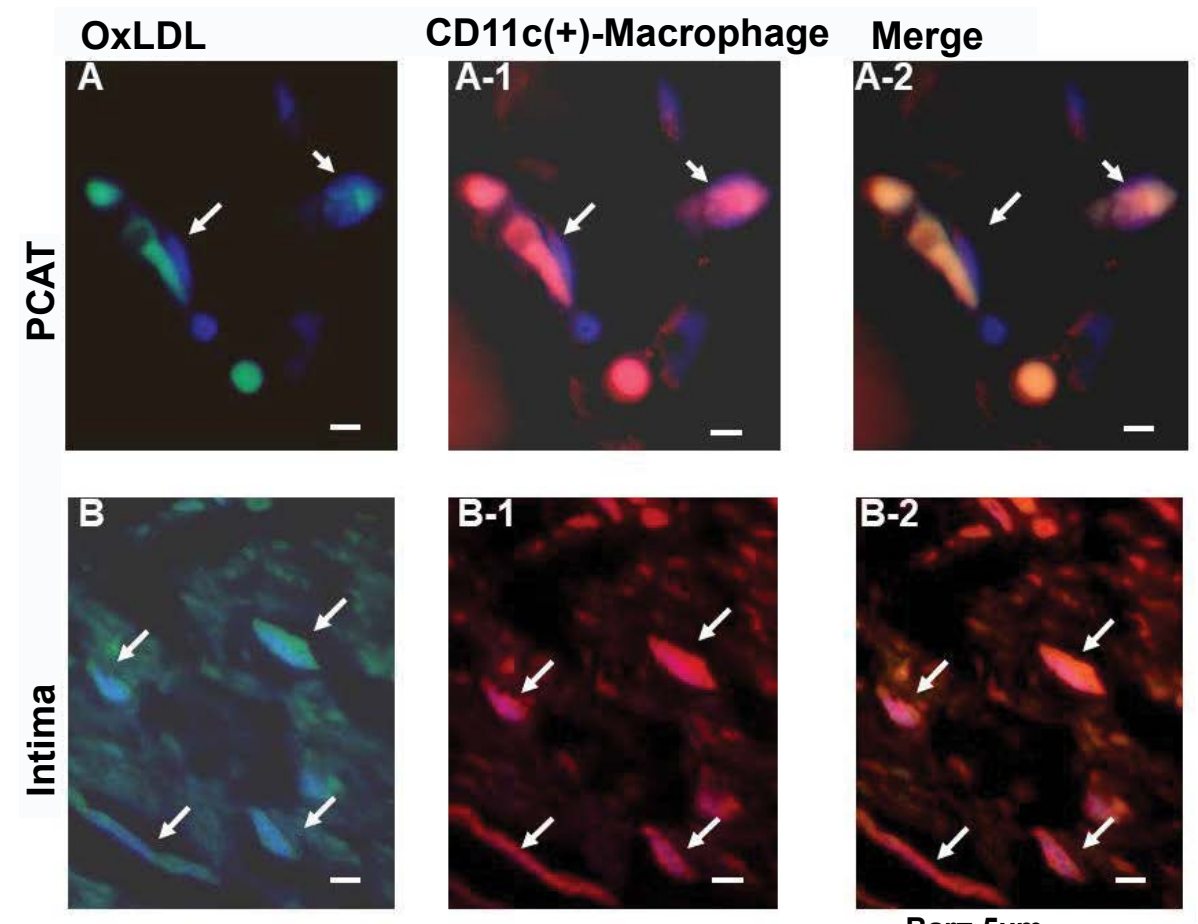

Figure 4: CD11c (+) macrophages (M1- Macrophage) that contain oxidized low-density lipoprotein (OxLDL). A - A2: CD11c(+)-macrophages which contain oxLDL in PCAT (arrows). B - B-2: CD11c(+) - macrophages which contain oxLDL in intima (arrows). Bar=5 $\mu \mathrm{m}$. 


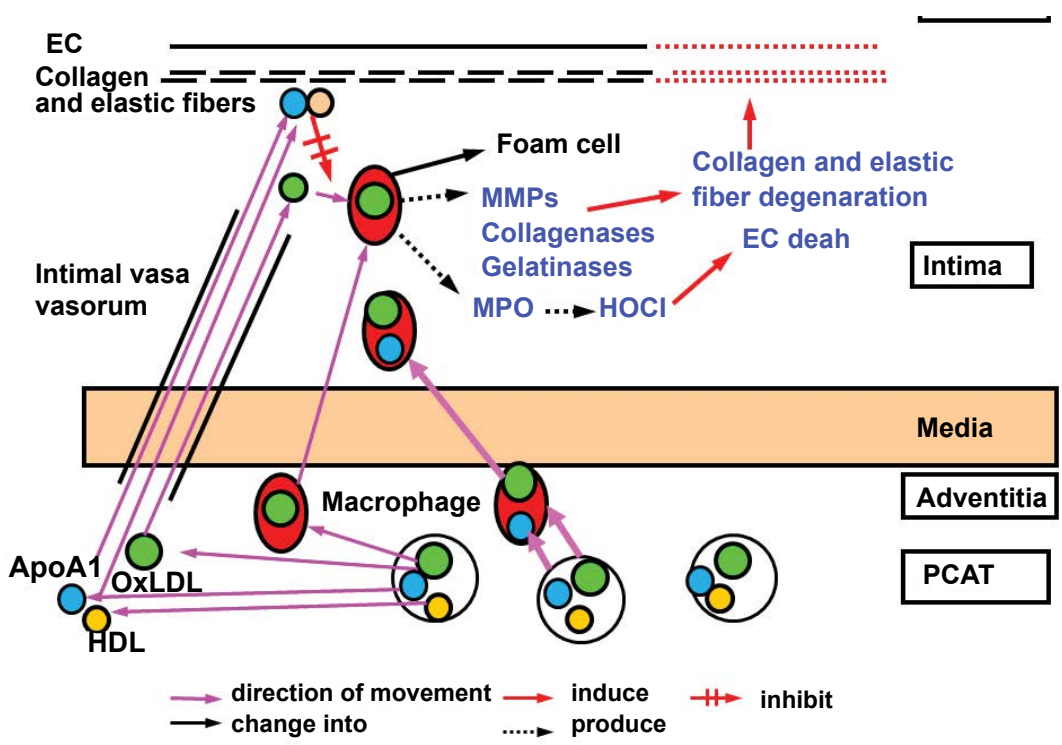

Figure 5: Possible mechanisms of the storage and supply of oxidized low-density lipoprotein (OxLDL), high-density lipoprotein (HDL) and apolipoprotein A1 (ApoA1) in human coronary intima (plaques). Schematic representation of a hitherto unrecognized supply route of oxLDL, HDL and ApoA1 to the coronary intima. They are stored in the PCAT, from where oxLDL and ApoA1 are conveyed by either CD68(+) macrophages or neovascularized vasa vasorum and HDL by vasa variorum. The macrophages accumulate oxLDL further and become foam cells while releasing matrix metalloproteases (MMPs) and collagenases, which in turn destroy collagen fibers/elastic fibers and also producing myeloperoxidase (MPO), which produces hypochlorous acid (HOCI) to destroy endothelial cells (EC), resulting in vulnerable plaque. HDL and possibly ApoA1 facilitate cholesterol uptake from cholesterol-loaded macrophage-foam cells in plaques for transport back to the liver, decrease oxLDL, increase nitric oxide synthesis and improve endothelial function.

ApoA1 was low, expanded with plaque growth and expanded further with plaque maturation as with HDL. This finding demonstrated that, despite an anti-atherogenic substance as with HDL, ApoA1 looked like oxLDL in the supply courses to the intima yet contrasted from oxLDL and resembled HDL in its connection to plaque morphology[16].

\section{LDL}

LDL deposits: Diffuse LDL deposits were found in the intima but not in PCAT (Figures 3A-1 and 3A-2). LDL co-localized with intimal vasa vasorum and its incidence in intima was low and had no undeniable connection to plaque morphology.

\section{Macrophage Phenotypes}

There are a number of macrophage phenotypes. Among them, M1macrophages are considered as atherogenic whereas M2- macrophages as anti-atherogenic, based mostly on the findings in animals or cultured cells.[17] To our knowledge, there are no reports on storage of oxLDL, HDL or ApoA1 in human PCAT. In our unpublished study, a certain group of CD11c (+) -macrophages (M1- macrophages) contained oxLDL or ApoA1 but not HDL (Figure 4). CD 206(+) - macrophages (M2-macrophages) that contain oxLDL or ApoA1 but not HDL were also found. The findings suggested that there are subspecies in both M1 and M2 macrophages in respect to lipoprotein or apolipoprotein carrying capacity and that there are no definite differences in carrying capacity of lipoproteins and apolipoproteins between M1 and M2 macrophages. Switching of M2 to M1 macrophages, which was demonstrated in animals [18], might have occurred in human macrophages and caused such phenomena. Further studies are necessitated to clarify the role of individual macrophage phenotypes in carrying these proteins.

\section{Supply of OxLDL, HDL and ApoA1 from PCAT to Coronary Plaques}

Figures 5 represents our speculation on the capacity and conceibable supply routes of oxLDL, HDL and ApoA1. Imbalanced content in the PCAT and their supply to the intima may decide the destiny of plaques.

\section{Other Reports}

Perivascular adipocytes (PVA) deliver substantial quantities of metabolically dynamic substances with both endocrine and paracrine actions [19]; PVA of human internal thoracic artery release NO [20]; PVA discharges nicotinamide adenine dinucleotide phosphateoxidase in mice [21]; rat PVA discharge compliment [23,22]; rat PVA discharge metylpalmitate ester [23]; different substances, for example, plasminogen activator inhibitor- 1 and interleukin- 6 are contained in epicardial adipose tissue in patients with acute coronary syndrome [24]; cultured human epicardial adipose tissues release glutation S-transferase P and ApoA1 [25]. But, reports depicting release and storage of lipoproteins from human PVA, including PCAT, to the nearby vessel wall were not found. Expanded volume of epicardial adipose tissue is a hazard factor for coronary heart disease [26,27]. Increased supply of pro-atherogenic lipoproteins from thick PCAT to coronary intima may quicken coronary atherosclerosis.

\section{Clinical Implications}

Our findings proposed that molecular therapy targeting lipoproteins in the PCAT could be successful in preventing coronary atherosclerosis.

\section{References}

1. Shah PK (2009) Inflammation and plaque vulnerability. Cardiovasc Drugs Ther 23: 31-40.

2. Chen JH, Riazzy M, Smith EM (2009) Oxidized LDL-mediated macrophage survival involves elongation factor-2 kinase. Arterioscler Thromb Vasc Biol 29: 92-98.

3. Holvoet P (2004) Oxidized LDL and coronary heart disease. Acta Cardiol 59 479-484.

4. Takahashi K, Takeya M, Sakashita N (2002) Multifunctional roles of macrophages in the development and progression of atherosclerosis in human 
Citation: Uchida Y, Uchida Y (2017) Pericoronary Adipose Tissue as Possible Supply Site and Storage for Lipoproteins in Human Coronary Artery. Mol Biol 6: 195. doi: 10.4172/2168-9547.1000195

Page 5 of 5

and experimental animals. Med Electron Microsc 35: 179-203.

5. Mann D, Zipes D, Libby P, Bonow R (2015) The vascular biology of atherosclerosis. In: Heart disease 10th Edition. Elsevier Saunders Ltd, Philadelphia, pp: 873-890.

6. Yoshida H, Kisugi R (2011) Mechanisms of LDL oxidation. Chimica Acta 411 1875-1882.

7. Assmann G, Schulte H, von Eckardstein A, Huang Y (1996) High-density lipoprotein cholesterol as a predictors of coronary heart disease risk. The PROCAM experience and pathophysiological implications for reverse cholesterol transport. Atherosclerosis 124: 11-20.

8. Toh PP, Davidson MH (2010) High-density lipoproteins: Marker of cardiovascular risk and therapeutic target. J Clin Lipidol 4: 359-364.

9. Cho KH (2009) Biomedical implications of high-density lipoprotein: its composition, structure, functions and clinical applications. BMB Rep 42: 393400 .

10. Boekholdt SM, Arsenault BJ, Hovingh GK, Mora S, Pedersen TR, et al. (2013) Levels and changes of HDL cholesterol and apolipoprotein A-1 in relation to risk of cardiovascular events among statin-treated patients: A meta-analysis. Circulation 128:1504-1512.

11. Emoto T, Sawada T, Morimoto N, Tenjin T, Wakimoto T, et al. (2013) The apolipoprotein B/A1 ratio is associated with reactive oxygen metabolites and endothelial dysfunction in statin-treated patients with coronary artery disease. $J$ Atheroscler Thromb 20: 623-629.

12. Pan L, Lu G, Chen Z (2014) Combined use of apolipoprotein B/apolipoprotein A1 ratio and non-high-density lipoprotein cholesterol before routine clinical lipid measurement in predicting coronary heart disease. Coron Artery Dis 25: 433-

13. Uchida Y, Uchida Y, Shimoyama E, HirutaN, Kishimoto T, et al. (2016) Pericoronary adipose tissue as storage and supply site for oxidized low-density lipoprotein in human coronary plaques. PLoS ONE 11: 0150862.

14. Uchida Y, Uchida Y, Shimoyama E, Hiruta N, Kishimoto T, Watanabe S, et al. (2017) Human pericoronary adipose tissue as storage and possible supply site for oxidized low-density lipoprotein and high-density lipoprotein in coronary artery. J Cardiol 69: 236-244.

15. Uchida $Y$ (2001) Clinical classification of atherosclerotic coronary plaques. In: Coronary angioscopy, Uchida Y (ed), Futura Publishing Ltd, Armonk, NY, pp: 71-81.
16. Uchida $Y$, Uchida Y, Shimoyama E (2017) Deposition patterns and localization of apolipoprotein $\mathrm{A} 1$ and their relation to plaque morphology in human coronary artery. JSM Atherosclerosis 2: 1025-1034.

17. DePaori F, Staeles B, Chinetti-Ghaguidi G (2014) Macrophage phenotypes and their modulation in atherosclerosis. Circ J 78: 1775-1781.

18. Kallou-Lachest J, Varthuaman A, Fornasa G, et al. (2010) Macriphage plasticity in experimental atherosclerosis. PLoS ONE 5: e8852.

19. Szasz T, Webb C (2010) Perivascular adipose tissue: More than just atructura support. Clin Sci 122: 1-12.

20. Malinowski M, Deja MA, Gołba KS, Roleder T, Biernat J, et al. (2008) Perivascular tissue of internal thoracic artery releases potent nitric oxide and prostacyclin-independent anticontractile factor. Eur J Cardiothoracic Surg 33: 225-231.

21. Ketonen J, Shi J, Mervaala E (2010) Priadventitial adipose tissue promotes endothelial dysfunction via oxidative stress in diet-induced obese C57BI/6 mice. Circ J 74: 1479-1487.

22. Ruan CC, Zhu DL, Chen QZ, Chen J, Guo SJ, et al. (2010) Perivascular adipose tissue-derived complement 23 is required for adventitial fibroblast functions and adventitial remodeling in deoxycorticosterone acetate-salt hypertensive rats. Arterioscler Thromb Vasc Biol 30: 2568-1574

23. Lee YC, Chang HH, Chiang CL, Liu CH, Yeh JI, et al. (2011) Role of perivascula adipose tissue-derived methyl palmitate in vascular tone regulation and pathogenesis of hypertension. Circulation 124: 1160-1171.

24. Langheim S, Dreas L, Veschini L, Maisano F, Foglieni C, et al. (2010) Increased expression and secretion of restin in epicardial adipose tissue of patients with acute coronary syndrome. Am J Physiol Heart Circ Physiol 298: 746-753.

25. Salgado-Somoza A, Teijeira-Fernández E, Fernández ÁL, González Juanatey JR, Eiras S, et al. (2012) Changes in lipid transport-involved proteins of epicardial adipose tissue associated with coronary artery disease. Atherosclerosis 224: 492-499.

26. Verhagen SN, Visseren FL (2011) Perivascular adipose tissue as a cause of atherosclerosis. Atherosclerosis 214: 3-10.

27. Shimabukuro M, Hirata Y, Tabata M, Dagvasumbere M, Sato H (2013) Epicardia adipose tissue volume and adipokine imbalance are strongly linked to human coronary atherosclerosis. Arterioscler Thromb Vasc Bio 33: 1077-1084.

28. Uchida $Y$ (2001) Clinical classification of atherosclerotic coronary plaques. In: Coronary angioscopy, Uchida $Y$ (ed), Futura Publishing Ltd, Armonk, NY, pp: 71-81 